\title{
EDITORIAL
}

\section{ECONOMETRIC THEORY AND PRACTICE}

\author{
Peter C.B. Phillips \\ Cowles Foundation for Research in Economics, Yale University \\ University of Auckland \\ University of York \\ and
}

Singapore Management University

Econometrics has been evolving as a discipline over the last decade in a way that has successfully brought theory and practice much closer together. Many of the developments are associated with laptop computing, the increasing availability of electronic databases, and the convenience of modern econometric software and matrix programming languages. The changes that have occurred affect us at every level as teachers, researchers, practitioners, readers, reviewers, and authors. No journal can stand still in the face of such changes. This editorial speaks to these changes and the way they impact our subject, our authors, and our readership.

In the classroom, practical aspects of implementing econometric procedures now figure prominently in expositions of econometric ideas. Simulations are conducted to show how econometric methods work and to reveal performance characteristics against competing procedures in finite samples, examples are used to produce results from empirical applications, and programming code is developed alongside theory to show how algebraic formulas translate into practical algorithms. Such is the practical skill set that the new generation of econometrician takes to the workplace.

In research, core theory in microeconometrics and time series econometrics has expanded rapidly, assimilating new models and structures, new approaches to estimation and inference, and new technologies for implementation and presentation. During the last decade these twin sisters of econometrics have grown closer in form and substance as models, methods, and concerns have become interrelated through the use of nonparametric methods and function space asymptotics, the need to address the complications of spatially and temporally dependent data, and the advances in multidimensional limit theory. 
Just as these two branches of econometrics have become increasingly interwoven, the connecting corridors that link econometrics to mathematics, statistics, engineering, and the applied and social sciences have grown wider, opening up new fields of application with fresh problems to resolve and new data sets to utilize in applied work. In all these developments, practical matters either sit at the forefront or are prominent behind the scenes in pushing forward the research agenda.

As computational methods have advanced, so too has the profession's interest in numerically intensive procedures such as the bootstrap, subsampling, empirical likelihood, indirect inference, and jackknifing. These methods are being used increasingly in practical work as we continue to explore their theoretical properties. Automated econometric procedures - autometrics - open up the possibility of conducting econometric modeling, estimation, and policy analysis in an automated way where data-based methods select suitable models, perform inference, and generate forecasts. Programs for the implementation of these methods have been developed and are being refined, and extensive developments may be expected in the future.

Alongside these changes, the subject of financial econometrics has exploded into the picture. Financial econometrics has played a strategic role in combining theory and practice. The subject is young and rapidly expanding in scope with strong connections to the finance and banking industries. Driven by huge advances in electronic databases, the vast growth in financial markets, the increasing complexity of financial products and services, and the demand for real-time financial analysis, the development of econometric tools for use in financial applications has mushroomed. The field is hardly two decades old, but its growing significance has already been recognized by the shared award of the Nobel Prize in Economics in 2003.

When the journal Econometric Theory was founded, one of its primary goals was to support innovative theoretical developments in econometrics. This objective was quickly realized, and preeminence in theory has long been the defining characteristic of the journal. Two decades ago, it was conventional for ET articles to develop new theory and say little about empirical application or practical implementation. Potential areas of application might be indicated, but sometimes the time horizon of implementation would be long term. Now, as ET celebrates a quarter century of publication, it is standard practice in a theory paper to include an empirical illustration, numerical simulations that investigate finite-sample performance, and some matters of practical implementation. Twenty years ago this research would likely have been spread across several papers and journals.

The changes have also affected the journal review process. Referees frequently request algorithms for implementation or empirical applications to demonstrate practicality. Reviewers sometimes implement the procedures suggested in a paper themselves and cross-check a paper's findings by simulation. Informed reviews and editorial nudges of this type have quietly helped to guide changes in the form that published econometric work takes. 
Of course, these changes have been facilitated by ease of computation and the advance of computer power to the laptop. Many papers make small, but nonetheless useful, theory advances and push the practical dimension forward in interesting ways that often lead to new and productive veins of theory. Time series econometrics abounds with examples from the two decades of development that have followed the unit root and cointegration revolution of the 1980s. Similarly, new econometric methods have emerged in financial econometrics to meet the needs of ultra-high-frequency data, in microeconometrics to address problems of weak instrumentation, in spatial econometrics to deal with issues of cross-section dependence, and in auction and industrial organization econometrics to resolve matters of identification. All these fields show an interfacing of theory and practice that is changing the landscape of modern econometrics.

Many of the changes described here are manifest in the articles now published in ET. One only needs to compare early issues of ET in the 1980 s with recent issues to see the evolution that has occurred. This evolution has broadened the scope of ET de facto, reflecting the reality of the present range and nature of the subject. We will better serve subject, authors, and readership in the future by acknowledging this closer interface of theory and practice and by encouraging submissions of work that further promotes this goal. Accordingly, the scope of ET is now explicitly broadened to encompass both econometric theory and practice. The change is mirrored in an updated Aims and Scope of the journal that appears in this issue on the inside back cover. The expansion of our ambit enables us to provide an outlet for new veins of theory, as we do at present, while at the same time encouraging good practical demonstration and implementation.

This expansion of ET broadens the scope of the journal in a way that reflects the current nature of econometrics. It does not convert ET into an applied journal or a journal that will publish a lot of empirical applications. We maintain our primary goal of supporting innovative theoretical developments in econometrics and continue to attract and encourage pure theory as our central business. At the same time, we can help to promote best practice econometrics by publishing work that demonstrates new theory and the practical implementation of theory. By fostering the growing relationship between theory and practice, ET recognizes that research developments run between theory and practice in both directions at the frontier of the subject. In short, good theory and practice often go hand in hand.

This issue of ET initiates the expansion in our goals by publishing a leading new paper in this genre of "econometric theory and practice." The paper is published complete with discussion by a panel of experts in the field, providing a focal point for further discussion and development. The paper is written by David Harvey, Stephen Leybourne, and Robert Taylor, and it addresses the practical issue of how best to conduct unit root testing. The topic has been a major area of research for econometricians over two decades and has important practical implications for the vast amount of applied work that relies on unit root tests. The subject is presently characterized by some divergence between theory and practice. Practice still relies heavily on augmented Dickey-Fuller (ADF) and 
Phillips-Perron tests, which were both developed in the 1980s at the outset of the unit root revolution. The dependence is explained by several factors: (i) convenience of the wide availability of these procedures in standard software packages; (ii) confusion over the multiplicity of alternative testing procedures, many of which do not lead to perceptibly important gains and which have their own various vulnerabilities; and (iii) practical anxiety over additional parameter settings that are required by some other procedures and misunderstandings about how best to implement them.

Two centrally important practical issues of unit root testing are addressed in the Harvey-Leybourne-Taylor (HLT) paper. These concern how to deal with the possible presence of a linear trend or drift in the data and how to manage uncertainty over the initial condition. The paper demonstrates the bearing of each of these issues on the performance of unit root testing and provides clear recommendations for practitioners about how to address these issues in practical work. The commentaries by Patrick Marsh, Ulrich Müller, Jörg Breitung, Peter Burridge, and Zhijie Xiao help to further clarify these practical matters of unit root testing, and they affirm the recommendations made in the HLT paper concerning best practice econometric methodology.

The HLT paper and its attendant commentaries demonstrate how the combination of econometric theory, empirical experience, and simulation evidence assists in revealing the path to good practical implementations of econometric methods. Future issues of ET will publish further articles and discussions on this interface between econometric theory and econometric practice. Our co-editors will work with me to achieve area balance so that various subfields and methodologies of econometrics are covered in these discussions.

ET welcomes your thoughts, ideas, and contributions on this innovation to strengthen this important ground between econometric theory and econometric practice. 